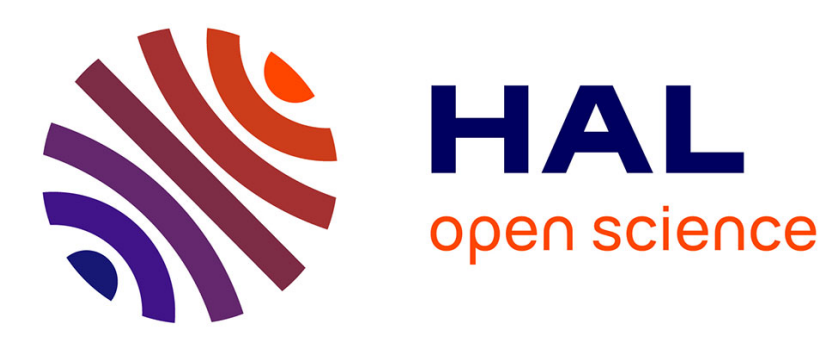

\title{
Contribution of Surface Distributions to Constant-Phase-Element (CPE) Behavior: 2. Capacitance
}

Christopher L. Alexander, Bernard Tribollet, Mark E. Orazem

\section{- To cite this version:}

Christopher L. Alexander, Bernard Tribollet, Mark E. Orazem. Contribution of Surface Distributions to Constant-Phase-Element (CPE) Behavior: 2. Capacitance. Electrochimica Acta, 2016, 188, pp.566573. 10.1016/j.electacta.2015.11.135 . hal-01255003

\section{HAL Id: hal-01255003 https://hal.sorbonne-universite.fr/hal-01255003}

Submitted on 13 Jan 2016

HAL is a multi-disciplinary open access archive for the deposit and dissemination of scientific research documents, whether they are published or not. The documents may come from teaching and research institutions in France or abroad, or from public or private research centers.
L'archive ouverte pluridisciplinaire HAL, est destinée au dépôt et à la diffusion de documents scientifiques de niveau recherche, publiés ou non, émanant des établissements d'enseignement et de recherche français ou étrangers, des laboratoires publics ou privés. 


\title{
Contribution of Surface Distributions to Constant-Phase-Element (CPE) Behavior: 2. Capacitance**
}

\author{
Christopher L. Alexander ${ }^{\mathrm{a}}$, Bernard Tribollet ${ }^{\mathrm{b}}$, Mark E. Orazem ${ }^{\mathrm{a}, *}$ \\ ${ }^{a}$ Department of Chemical Engineering, University of Florida, Gainesville, FL, 32611, \\ USA \\ ${ }^{b}$ LISE, UPR 15 du CNRS, Université P. et M. Curie, CP 133, 4 Place Jussieu, 75252 \\ Paris cedex 05 France
}

\begin{abstract}
Finite-element simulations were performed to determine the influence of a radial distribution of capacitance on the impedance of ideally polarized disk electrodes. The characteristic length associated with this form of distribution was found to be the period of the distribution. This work showed that while a capacitance distribution causes frequency dispersion, this effect is seen only at frequencies that are much higher than those associated with the disk-geometry-induced frequency dispersion. Thus, a constant-phaseelement associated with a surface distribution of time constants cannot be attributed to a distribution of capacitance.
\end{abstract}

Keywords: Frequency Dispersion, capacitance, impedance spectroscopy

\section{Introduction}

Impedance measurements on solid electrodes often yield constant-phaseelement(CPE) behavior over a large range of frequencies. A CPE describes a distribution of time constants which can be physically categorized as oc-

\footnotetext{
*Corresponding author

Email address: meo@che.ufl.edu (Mark E. Orazem)
}

${ }^{* *}$ published in Electrochimica Acta, 188 (2016), 566-573. 
curring either normal to or along the electrode surface [1].

Hirschorn et al. $[2,3]$ used a measurement model incorporating a series of elements to show that a power-law distribution of resistivity through an oxide film gives rise to $\mathrm{CPE}$ behavior. This model has been used to determine the film thickness of oxides on stainless steel and aluminum electrodes[4]. It has also been used to determine physical properties of human skin[4,5] and polymer coatings $[6]$.

Distributions of solution resistance are observed in planar disk electrode experiments. Huang et al. showed that the geometry of a disk electrode within an insulating plane leads to frequency dispersion on blocking electrodes at frequencies greater than dimensionless frequency $\omega C_{0} r_{0} / \kappa=1$ [7]. Jorcin et al. [1] confirmed these results experimentally with the use of local electrochemical impedance spectroscopy.

Surface roughness of the electrode, once believed to contribute to the cause for frequency dispersion [8], will also yield a distribution of ohmic resistance. Alexander et al.[9] showed that surface roughness at the micron scale leads to frequency dispersion at frequencies larger than those due to the disk geometry if the radius of the disk is greater than the characteristic length, $f_{r} P$, where $f_{r}$ is the roughness factor and $P$ is the period of the roughness. The period of the roughness may represent the average width of the rough grooves. The disk radius will in most cases be greater than this characteristic dimension except in cases of very large roughness factors such as those that yield porous electrode behavior. This result contradicts the theory that surface roughness may be a source of CPE behavior over a large range of frequencies.

Another proposed cause of frequency dispersion is the surface distribution of capacitance [10]. Brug et al. developed a formula which relates the 
parameters of the CPE to an effective double layer capacitance [11]. They postulated that the distribution was due to a variation in capacitance across the electrode surface.

In 1978, Leek and Hampson [10] calculated the frequency dispersion using a circuit element ladder with different capacitance values per surface area of electrode. They concluded that surface heterogeneity was an important factor contributing to frequency dispersion. In 1992, Pajkossy and Nyikos [12] used a self-similar capacitance distribution to show that frequency dispersion in the form of constant-phase-element behavior cannot be explained by a capacitance distribution since the CPE behavior is only observed for physically impossible variations in capacitance. Also in 1992, Kurtkya and de Levie [13] conducted numerical simulations to explain that the frequency dispersion due to nonuniform capacitance is caused by the shift of the current lines with frequency from locations of high impedance to locations of low impedance.

The objective of this work is to explore, by use of finite-element models, whether a surface distribution of capacitance could provide a valid physical explanation for $\mathrm{CPE}$ behavior over a broad range of frequencies and to determine the characteristic frequency and dimension associated with this form of surface heterogeneity.

\section{Impedance Calculations}

Two disk electrode configurations were simulated, including a disk electrode embedded within an insulating plane, and a recessed disk electrode. The calculations were performed in COMSOL Multiphysics 4.3 using cylindrical coordinates for a quarter of a circle domain, provided in Figure 1(a), 


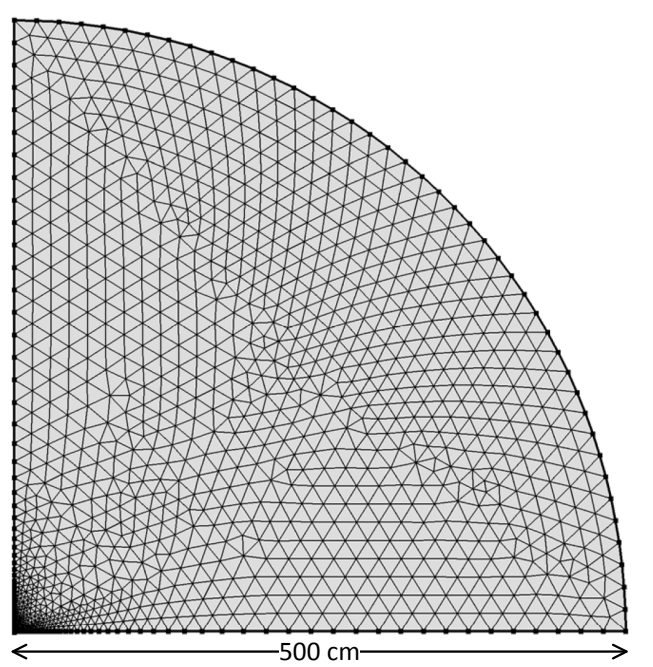

(a)

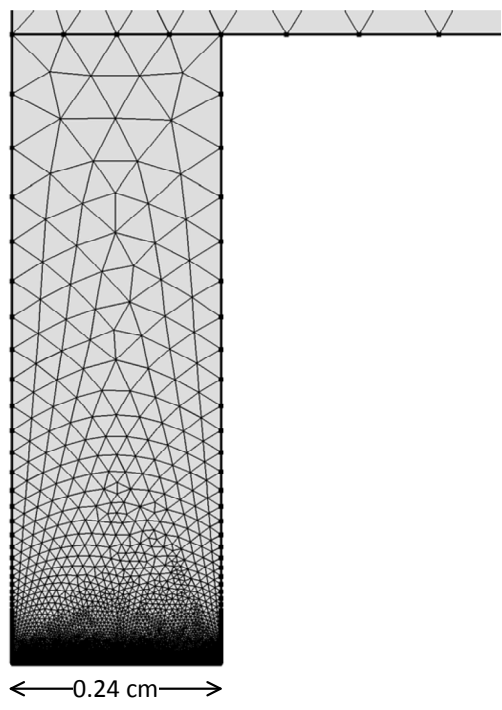

(b)

Figure 1: Finite-element mesh for the disk electrode simulations. a) Axi-symmetric domain used to represent disk electrode experiments; b) mesh showing a detailed view of a recessed electrode. The outer domain is shown in Figure 1(a). 


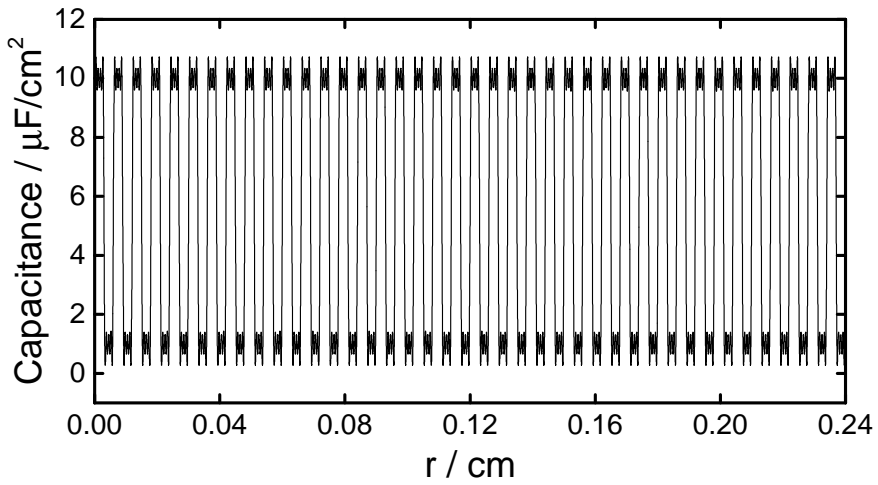

Figure 2: Capacitance distribution as a function of radial position based on a square wave represented by a Fourier series with a period of $60 \mu \mathrm{m}$.

which represented the electrolyte. Calculations were performed for electrode radii of $0.12 \mathrm{~cm}, 0.24 \mathrm{~cm}$, and $0.48 \mathrm{~cm}$. The counterelectrode was located at $\sqrt{r^{2}+y^{2}}=500 \mathrm{~cm}$, making the domain size at least 1000 times larger than the radius of the disk (see Alexander et al.[9]). This geometry was chosen to ensure that the counterelectrode could be considered to be infinitely far from the working electrode. A free triangular mesh was used with a greater density of elements near the working electrode.

A capacitance distribution was simulated by a Fourier series that represented a square wave distribution, as shown in Figure 2 as a function of radial position. The Fourier series[14] was expressed as

$$
C_{0}(r)=\langle C\rangle+\sum_{n=1}^{\infty}\left(C_{\max }-C_{\min }\right) \cos (2 n \pi r / P)
$$

where the constants $C_{\max }$ and $C_{\min }$ represented the maximum and minimum capacitance. The average capacitance of the electrode surface $\langle C\rangle$ was calculated as

$$
\left\langle C_{0}\right\rangle=\frac{2}{r_{0}^{2}} \int_{0}^{r_{0}} C_{0} r d r
$$


where $r_{0}$ is the radius of the disk and $C_{0}$ is given by equation (1). The capacitance as a function of the radial position was integrated over the surface of the electrode and then divided by the area of the electrode. The period $P$ of the square wave may be representative of an elemental grain size in which the capacitance is assumed to be relatively uniform across a grain and then jump to another value at an adjacent grain. Simulations were performed with 5, 10, 20, and 40 terms of the Fourier series. Numerical artifacts were observed at high frequencies when the series was truncated at 5 and 10 terms. As more terms were added to the series the artifacts shifted to higher frequencies and with 20 terms in the series this behavior was eliminated from the simulated frequency range. The Fourier series was therefore truncated after 20 terms.

The electrode was assumed to be purely capacitive, i.e., the contributions of slow reaction kinetics and mass-transfer were neglected. The potential distribution within the electrolyte domain was solved using Laplace's equation

$$
\nabla^{2} \Phi=0
$$

The potential comprises steady-state and oscillating components and may be expressed as

$$
\Phi=\bar{\Phi}+\operatorname{Re}\{\tilde{\Phi} \exp (\mathrm{j} \omega t)\}
$$

The potential of the electrode surface may also be expressed in the same manner as

$$
V=\bar{V}+\operatorname{Re}\{\tilde{V} \exp (\mathrm{j} \omega t)\}
$$

The normal oscillating current density at the surface of the electrode was expressed as

$$
\tilde{i}=\mathrm{j} \omega C_{0}(r)(\tilde{V}-\tilde{\Phi})
$$


where $\tilde{V}$ is the potential perturbation at the electrode and $\tilde{\Phi}$ is the complex oscillating potential within the electrolyte. The value of the potential perturbation will not affect the impedance response, but, for all of the stimulations presented in this work, a value of $10 \mathrm{mV}$ was used. The oscillating potential was constrained to be equal to zero at the counterelectrode. The impedance was calculated as

$$
Z(\omega)=\frac{\tilde{V}}{\tilde{i}}
$$

for a specified range of frequencies.

Frequency dispersion in impedance measurements may occur for blocking electrodes that yield a nonuniform current distribution across the working electrode surface. A dimensionless frequency $K$ may be expressed as the ratio of ohmic resistance and interfacial impedance as

$$
K=\omega C_{0} R_{\mathrm{e}}
$$

such that frequency dispersion is induced at $K \geq 1$. In the case of a blocking disk electrode within an insulating plane, the ohmic resistance in units of $\Omega \mathrm{cm}^{2}$ is given by[15]

$$
R_{\mathrm{e}}=\frac{\pi r_{0}}{4 \kappa}
$$

such that the dimensionless frequency is

$$
K=\frac{\pi}{4} \frac{\omega C_{0} r_{0}}{\kappa}
$$

The frequency at which dispersion occurs is found at $\mathrm{K}=1$ as

$$
f_{c}=\frac{2 \kappa}{\pi^{2} C_{0} r_{0}}
$$

Equation 11 may be used to estimate the frequency at which the disk geometry will begin to influence the impedance response. 


\section{Results and Discussion}

Impedance simulations are presented to show the influence of a heterogeneous surface capacitance on disk electrodes. Results are shown first for a recessed electrode configuration and then a disk electrode within an insulating plane. Dimensionless results are used to determine the characteristic lengths and frequencies associated with a radially-periodic distribution of capacitance.

\subsection{Capacitance Distribution on Recessed Electrodes}

A recessed electrode model was used to isolate the effect of surface heterogeneity on the impedance response of blocking electrodes. The simulated impedance response of a recessed disk electrode with the square wave capacitance distribution shown in Figure 2 is presented in Figure 3 as a function of frequency with the period of distribution as a parameter. The minimum and maximum capacitance values expressed in Equation 1 were set to $1 \mu \mathrm{F} / \mathrm{cm}^{2}$ and $10 \mu \mathrm{F} / \mathrm{cm}^{2}$ respectively to represent a surface with an oxide film and a bare metal surface. The conductivity of the solution was $10^{-5} \mathrm{~S} / \mathrm{cm}$. The impedance was representative of an ideal capacitor at frequencies below $100 \mathrm{kHz}$, shown by the vertical lines that are perpendicular to the real axis. The figure inset shows a magnified view of the impedance at high frequencies. As the period of the capacitance distribution increases, the frequency at which dispersion begins decreases.

The simulated real impedance as a function of frequency is presented in Figure 4(a) with the period of the capacitance distribution as a parameter. The low-frequency limit of the real impedance increased as the period of the distribution increased. The high-frequency limit, which represents the ohmic resistance, was unaffected by the distribution of capacitance. 


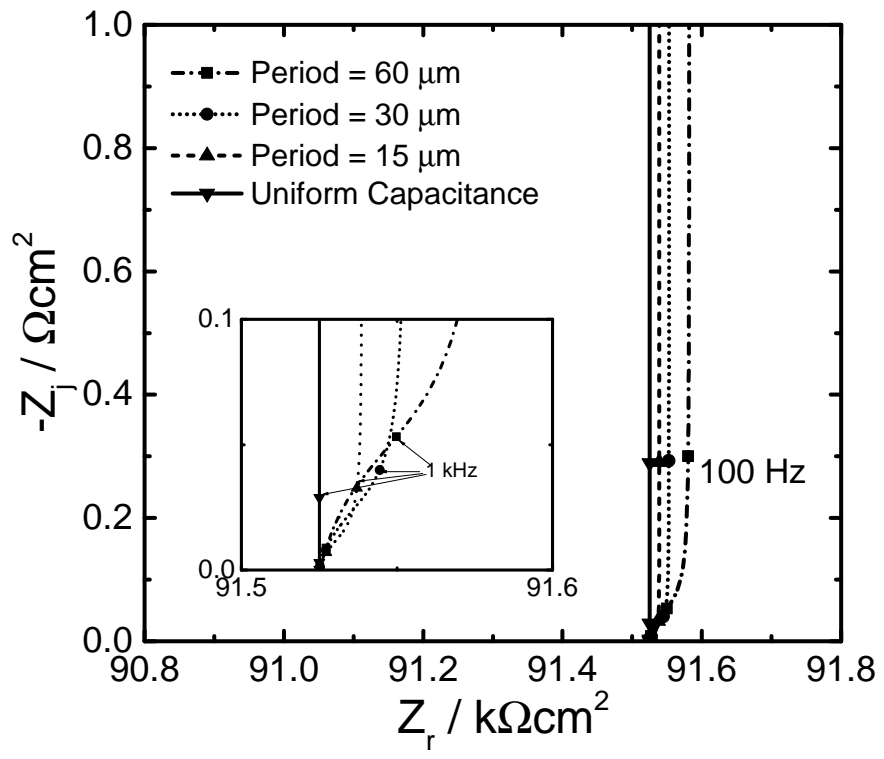

Figure 3: The impedance in Nyquist format of a recessed disk electrode with the square wave capacitance distribution shown in Figure 2 and the period of distribution as a parameter. 


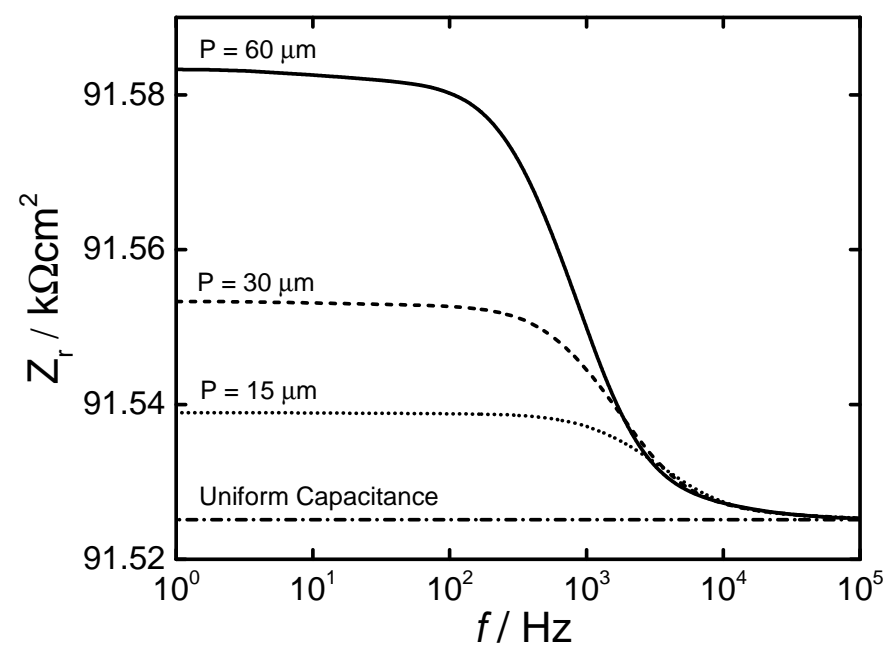

(a)

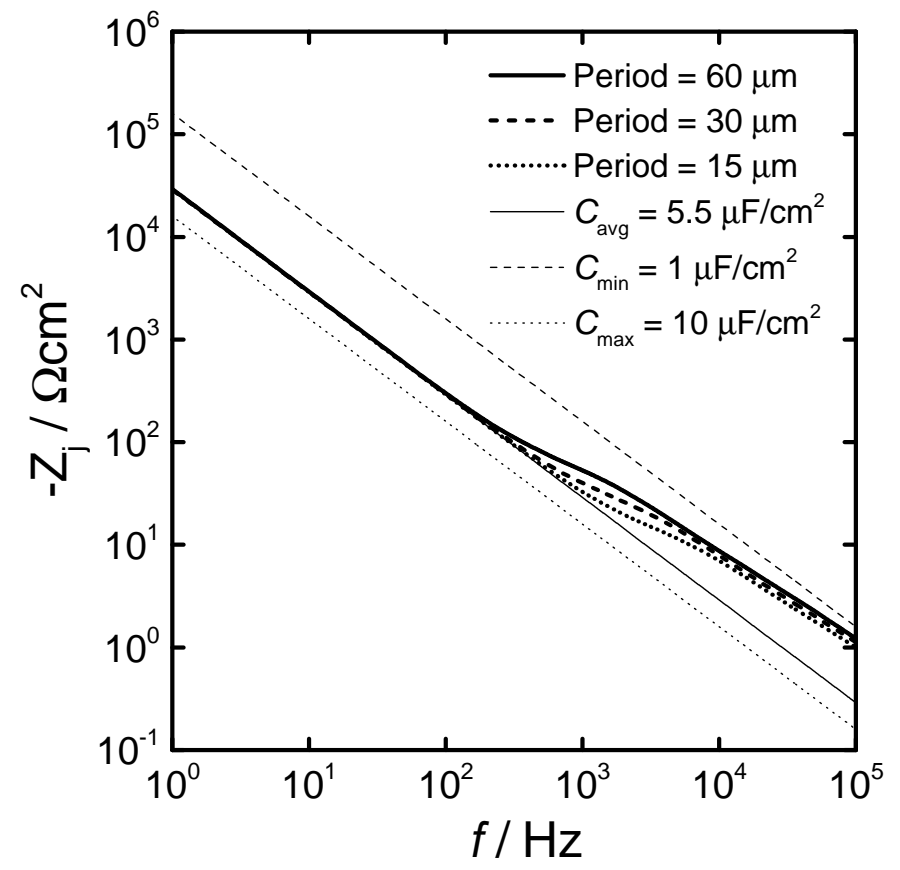

(b)

Figure 4: The simulated impedance as a function of frequency of a recessed disk electrode with a square wave capacitance distribution and the period of distribution as a parameter: a) the real part of the impedance; b) the imaginary part of the impedance. 
The imaginary impedance may be expressed as a function of the surfaceaveraged capacitance as

$$
\langle Z\rangle=\frac{-1}{\omega\left\langle C_{0}\right\rangle}
$$

Similar expressions may be formed for the maximum and minimum values of the capacitance. The impedance calculated from surface-averaged as well as the maximum and minimum capacitance values are compared to the simulated imaginary impedance in Figure 4(b) as a function of frequency with the period of the distribution as a parameter. The imaginary impedance at low frequencies coincided with the impedance based on the surface-averaged capacitance. Deviation from the surface-averaged impedance occurred at high frequencies where the impedance asymptotically approached the impedance associated with the minimum capacitance value.

The deviation from the expected impedance response may be explained by the current and potential distribution along the electrode surface, which is presented in Figure 5. In the case of blocking electrodes, the solution resistance controls the current distribution at high frequencies while the interfacial impedance controls at low frequencies. The potential distribution at low frequencies is represented by the color gradient in Figure 5(a). The streamlines corresponded to the path of the modulus of the oscillating current expressed, for a given frequency, as

$$
|\tilde{i}|=\sqrt{{\tilde{i_{\mathrm{r}}}}^{2}+\tilde{i}_{\mathrm{j}}^{2}}
$$

in which $\tilde{i_{\mathrm{r}}}$ and $\tilde{i_{\mathrm{j}}}$ represent the real and imaginary parts of the oscillating current. The false color representation of potential distribution was adjusted to emphasize the variations near the electrode surface. The regions of the electrode surface with higher capacitance have a lower impedance and the 


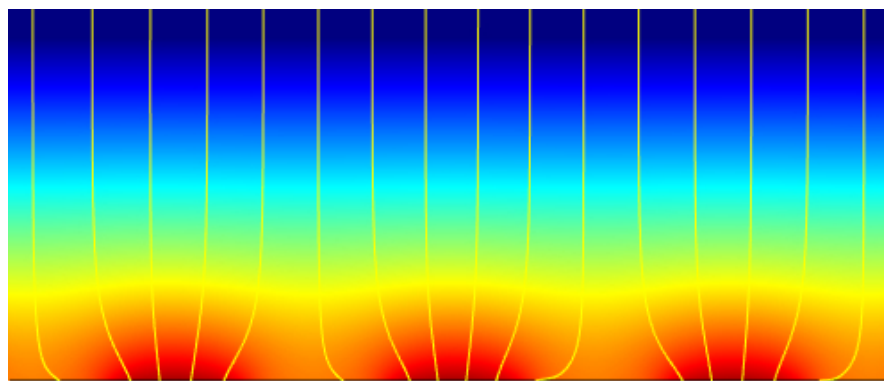

(a)

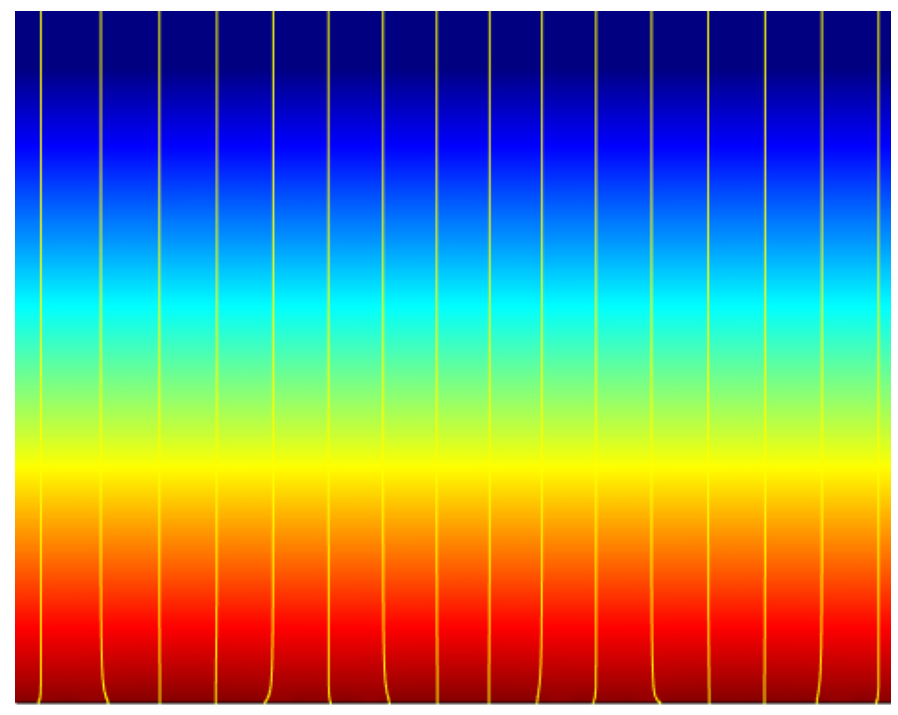

(b)

Figure 5: The current paths near the surface of a recessed electrode exhibiting a squarewave distribution of capacitance obtained as $\sqrt{\tilde{i}_{r}^{2}+\tilde{i}_{j}^{2}}$ : a) at $10 \mathrm{mHz}$; b) at $100 \mathrm{kHz}$. The potential distribution within the electrolyte adjacent to the rough surface is shown by the false-color representation. 
current flows more easily through these points. Despite the nonuniform current distribution, the impedance response at low frequencies was indicative of a pure capacitor with a value of the averaged surface capacitance. The potential distribution along the electrode surface at $100 \mathrm{kHz}$, presented in Figure 5(b), was more uniform.

The modulus of the oscillating current density along the electrode surface is presented in Figure 6 as a function of radial position. The low-frequency current response, presented in Figure 6(a), showed a variation of current proportional to the variation in surface capacitance and small values of current. The high frequency response, presented in Figure 6(b), showed much higher values of current however the distribution was much more uniform with only variations at locations where the capacitance changes from one value to the other which can be attributed to the finite number of terms in the Fourier series.

A phase angle dependent only on the imaginary part of the impedance was defined by Alexander et al. [9] as

$$
\varphi_{\mathrm{dZj}}=\frac{\operatorname{dlog}\left(Z_{\mathrm{j}}\right)}{\operatorname{dlog}(f)} \times 90^{\circ} .
$$

As compared to other definitions of phase angle, the imaginary-impedancederived phase angle is more sensitive to the onset of frequency dispersion. The imaginary-impedance-derived phase angle is presented in Figure 7(a) as a function of frequency with the period of distribution as a parameter. The effect of a varying capacitance along the surface of the electrode did not influence the imaginary impedance at low frequencies as indicated by a phase angle, $\phi_{d Z j}=-90^{\circ}$. However, frequency dispersion did occur in all cases at high frequencies with a minimum phase angle of approximately $-50^{\circ}$. The deviation from the expected capacitive behavior occurred at 


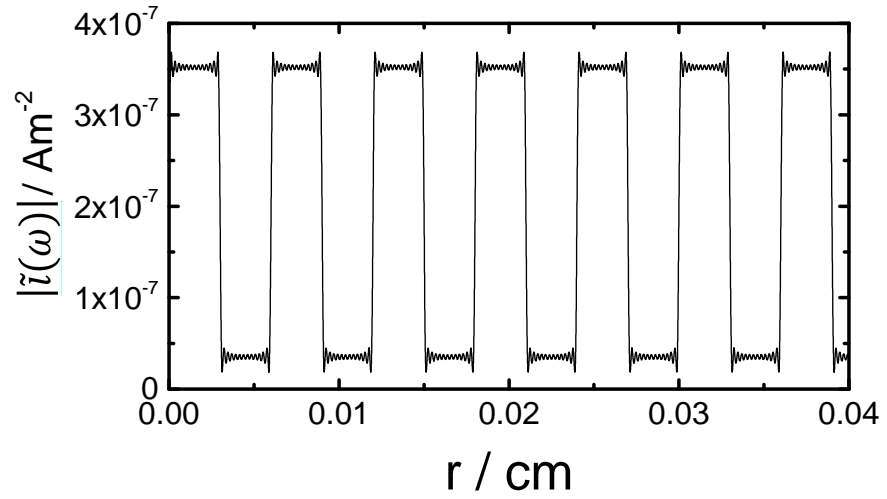

(a)

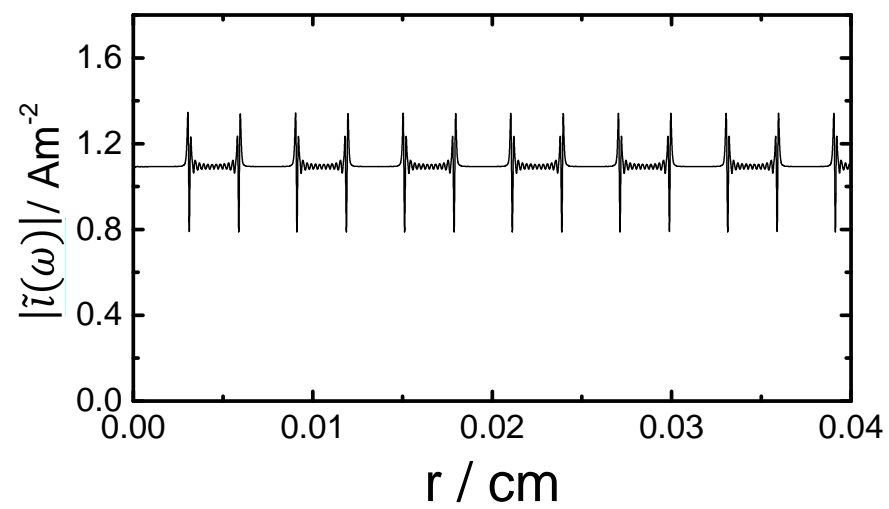

(b)

Figure 6: Normal current distribution at the electrode surface due to a nonuniform capacitance distribution with a period of $60 \mu \mathrm{m}$ of a recessed electrode as a function of radial position: a) current distribution at $10 \mathrm{mHz}$; b) current distribution at the high frequency limit of the simulations $f=100 \mathrm{kHz}$. 


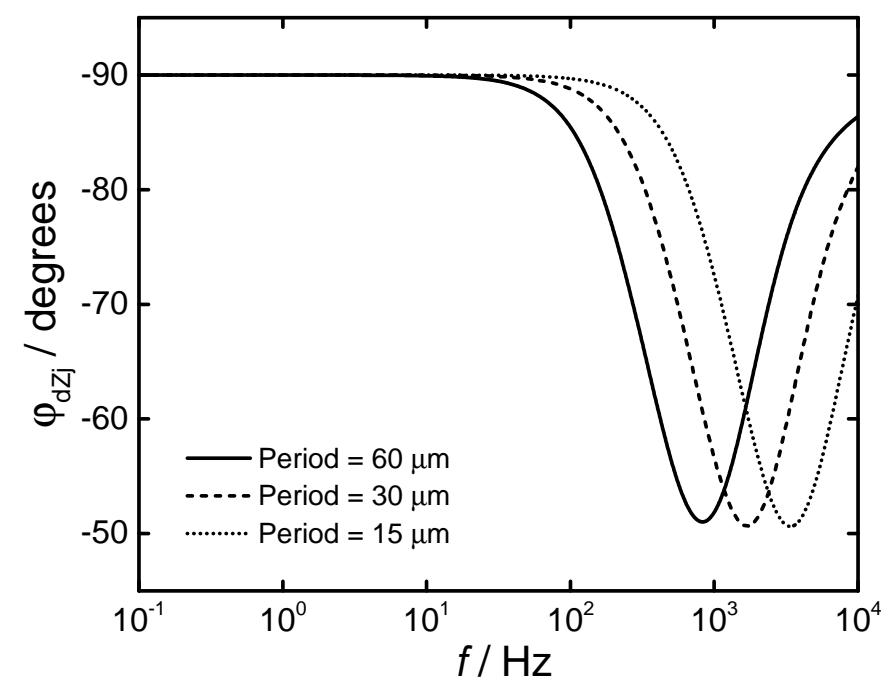

(a)

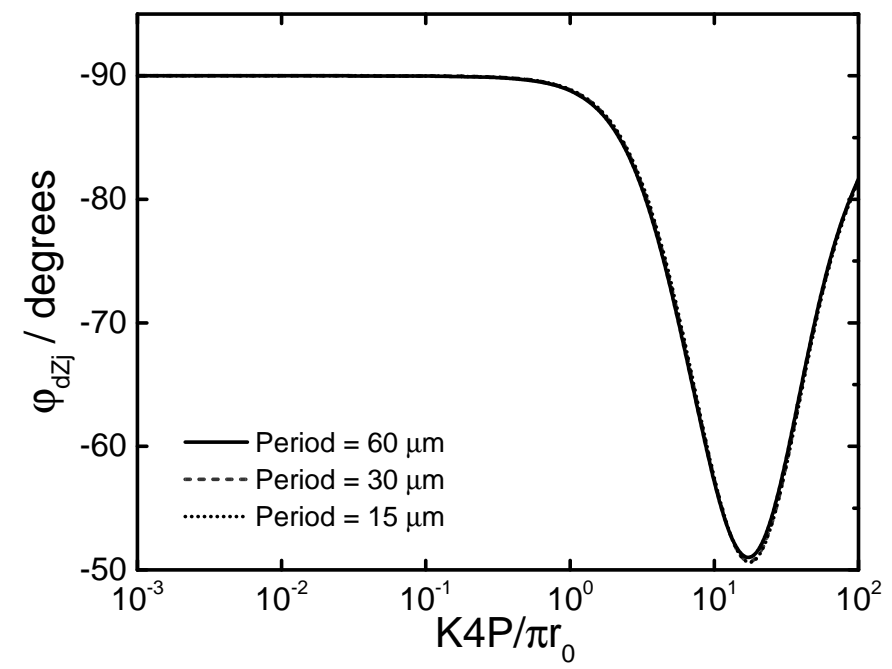

(b)

Figure 7: Imaginary-impedance-derived phase angle calculated from the impedance data in Figure 4: a) phase angle as a function of frequency; b) phase angle as a function of dimensionless frequency based on the averaged capacitance and the period of the distribution. 
lower frequencies as the period of the distribution increased. If the period of the distribution is taken as the grain size within a polycrystalline surface, grain sizes on the order of $1 \mu \mathrm{m}$ and less should not lead to frequency dispersion at frequencies less than $1 \mathrm{kHz}$.

A dimensionless frequency can be expressed for the distribution of capacitance by amending Equation 8 to include the surface-averaged capacitance expressed as

$$
K=\omega\left\langle C_{0}\right\rangle R_{\mathrm{e}, \mathrm{C}}
$$

where $R_{e, C}$ represents the ohmic resistance associated with the nonuniform capacitance distribution. The ohmic resistance associated with the capacitance distribution is proportional to the difference between the asymptotic values of the real impedance at high and low frequency. The ohmic resistance per unit area associated with a radially distributed capacitance may be approximated as

$$
R_{\mathrm{e}, \mathrm{C}} \approx \frac{P}{\kappa}
$$

where $P$ represents the period of the distribution. According to Equation 10, the dimensionless frequency may now be expressed as

$$
K 4 P / \pi r_{0}=\frac{\omega\left\langle C_{0}\right\rangle P}{\kappa}
$$

The impedance was scaled by the approximated ohmic resistance as $Z \kappa / P$. The imaginary-impedance-derived phase angle is presented in Figure 7(b) as a function of dimensionless frequency. The results were superposed, confirming that the period is the appropriate characteristic length to use to describe surface heterogeneity of capacitance. The characteristic frequency at which a non-uniform capacitance began to influence the impedance was determined to be $K 4 P / \pi r_{0}=1$. 


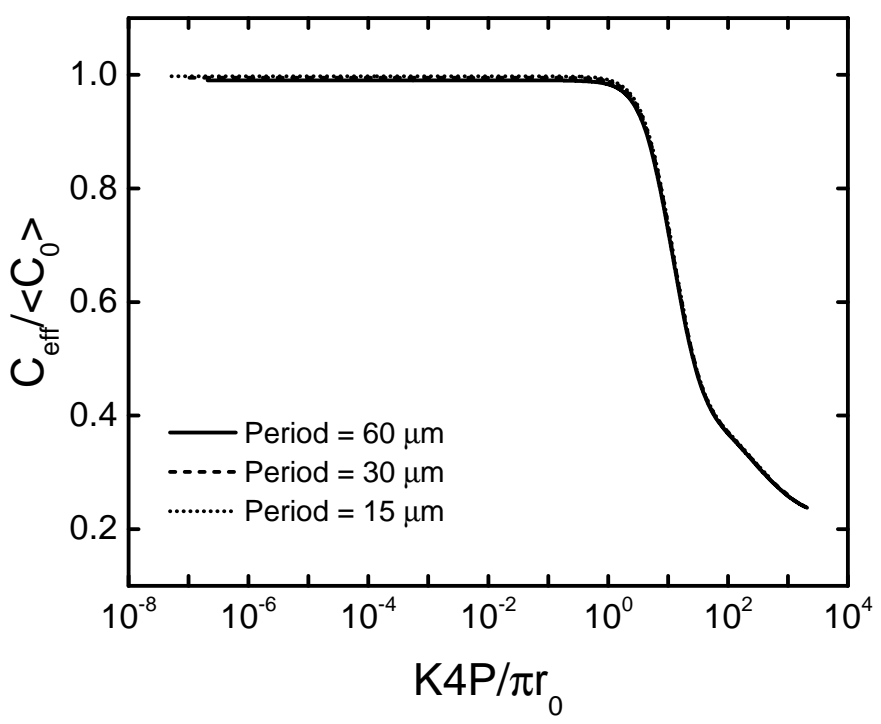

Figure 8: The ratio of the calculated effective capacitance and the surface-averaged input capacitance as a function of dimensionless frequency $K 4 P / \pi r_{0}$ with the period of the distribution as a parameter for recessed disk electrodes.

Since impedance is often used to measure interfacial capacitance, it is important to ensure that a distribution of capacitance along the surface does not complicate the use of this technique. The effective capacitance of the electrode/electrolyte interface may be determined from the imaginary part of the impedance as

$$
C_{\mathrm{eff}}=\frac{-1}{\omega Z_{\mathrm{j}}}
$$

The ratio of the calculated capacitance and the surface-averaged capacitance is shown in Figure 8 as a function of dimensionless frequency with the period of the distribution as a parameter. In all cases, the simulated capacitance closely matched the surface-averaged capacitance of the electrode at low frequencies. 


\subsection{Capacitance Distribution on Disk Electrodes}

The impedance of a disk electrode within an insulating plane containing a heterogenous surface capacitance was simulated to show the coupled effect of capacitance distribution and non-uniform current distributions due to disk geometry. The goal of these simulations was to determine if the effect of capacitance distribution influences the impedance at frequencies lower than the effect of disk geometry.

The geometry of a disk electrode embedded within an insulating plane causes frequency dispersion at high frequencies due to the non-uniform current distribution at high frequency. As the frequency increases to infinity, the magnitude of the oscillating component of the modulated current at the periphery of the disk approaches infinity while the current at the center of the disk remains finite. The simulated current distribution at an electrode surface containing the capacitance distribution in Figure 2 is provided as a function of radial position for $10 \mathrm{mHz}$ in Figure 9(a) and for $100 \mathrm{kHz}$ in Figure 9(b). The current distribution at low frequencies resembled the square-wave distribution features. At high frequencies, the effect of the disk geometry overshadowed the effect of the capacitance distribution since the current at the periphery approached infinity.

The imaginary-impedance-derived phase angle is provided in Figure 10(a) as a function of frequency. At low frequencies the phase angle was constant with a value equal to $-90^{\circ}$. Frequency dispersion became apparent at approximately $1 \mathrm{~Hz}$ for all periods of capacitance distribution due to the geometry of the disk. At higher frequencies, dispersion due to the nonuniform capacitance distribution occurred such that increases in the period of the distribution caused the deviation to shift to lower frequencies. The same results are presented in Figure 10(b) as a function of dimensionless 


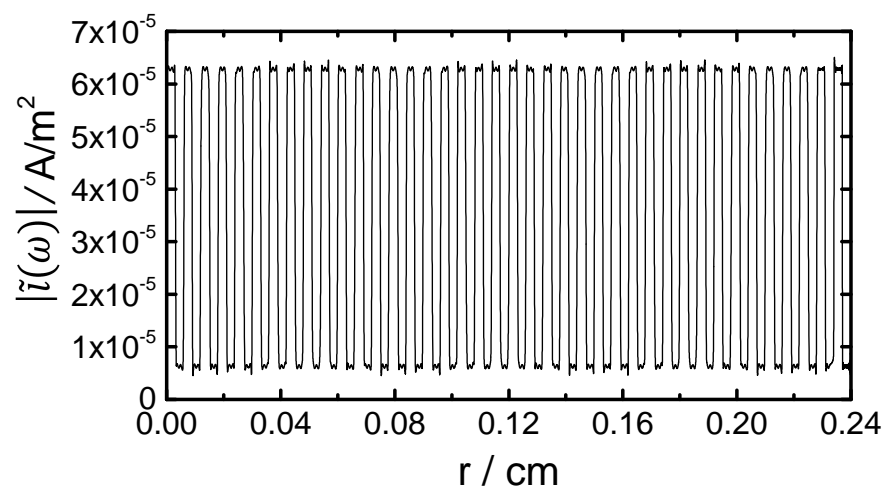

(a)

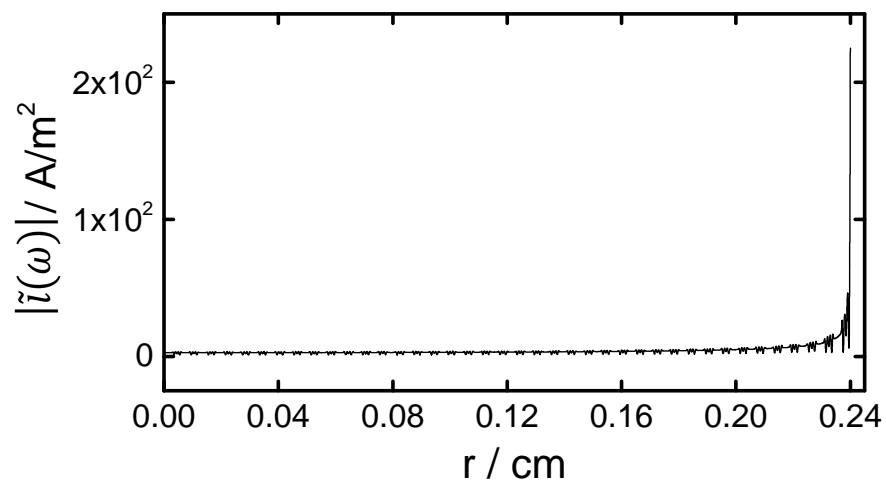

(b)

Figure 9: Normal current distribution at a disk electrode surface as a function of radial position: a) current distribution at $10 \mathrm{mHz}$; b) current distribution at $100 \mathrm{kHz}$. 


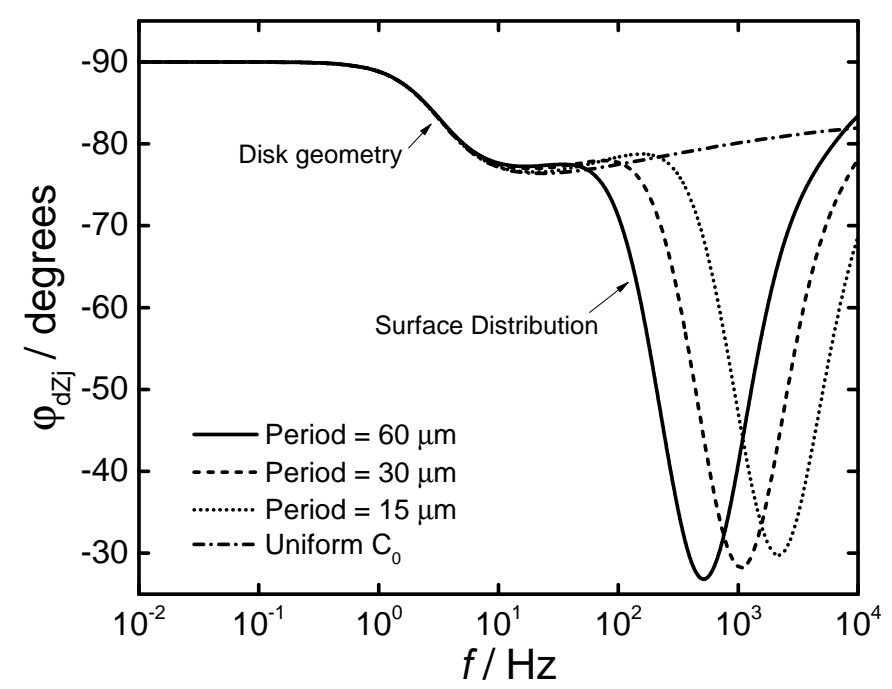

(a)

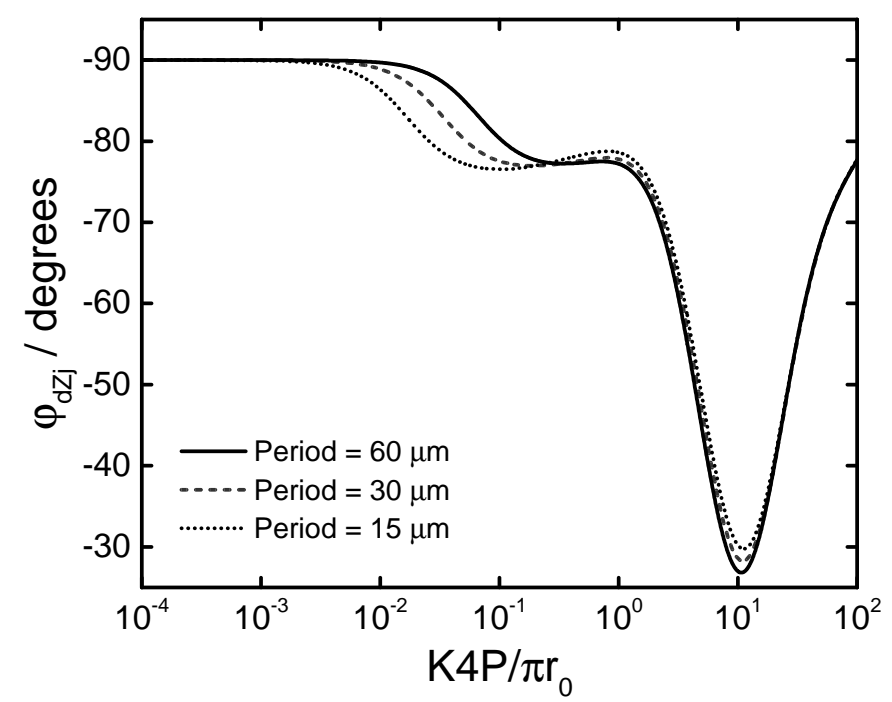

(b)

Figure 10: Imaginary-impedance-derived phase angle for a disk electrode within an insulating plane: a) phase angle as a function of frequency; b) phase angle as a function of dimensionless frequency based on the averaged capacitance. 
frequency in which the period of the distribution was used as the characteristic length. The frequency dispersion due to the surface heterogeneity was superposed, indicating that the period of the capacitance distribution is the appropriate characteristic length. The results obtained for a recessed electrode provided in Figure 7 show only the frequency dispersion due to the surface distribution of capacitance.

The imaginary-impedance-derived phase angles are presented in Figure 11(a) as a function of frequency with the radius of the disk as a parameter. The period of the distribution was fixed at $60 \mu \mathrm{m}$. Changes in the radius of the electrode influenced only the frequency dispersion associated with the geometry of the disk. The imaginary-impedance-derived phase angle is shown in Figure 11(b) as a function of dimensionless frequency, Equation 10. The frequency dispersion associated with disk geometry was superposed and the frequency dispersion associated with the capacitance distribution was not.

Simulations were performed to explore whether the amplitude of the capacitance distribution may influence the frequency at which the impedance is influenced. The imaginary-impedance-derived-phase angle was calculated from simulations on planar disk electrodes with different amplitudes of capacitance distribution and are presented in Figure 12 as a function of frequency. As the amplitude of the distribution increased, the deviation from $-90^{\circ}$ shifted to lower frequencies. However, when the frequency is made dimensionless with the use of the period as the characteristic length and the average value of the capacitance, shown in Figure 12(b), the characteristic frequency at which dispersion occurred did not change with amplitude and only the magnitude of the dispersion increased.

The ratio of the calculated capacitance and the average capacitance is 


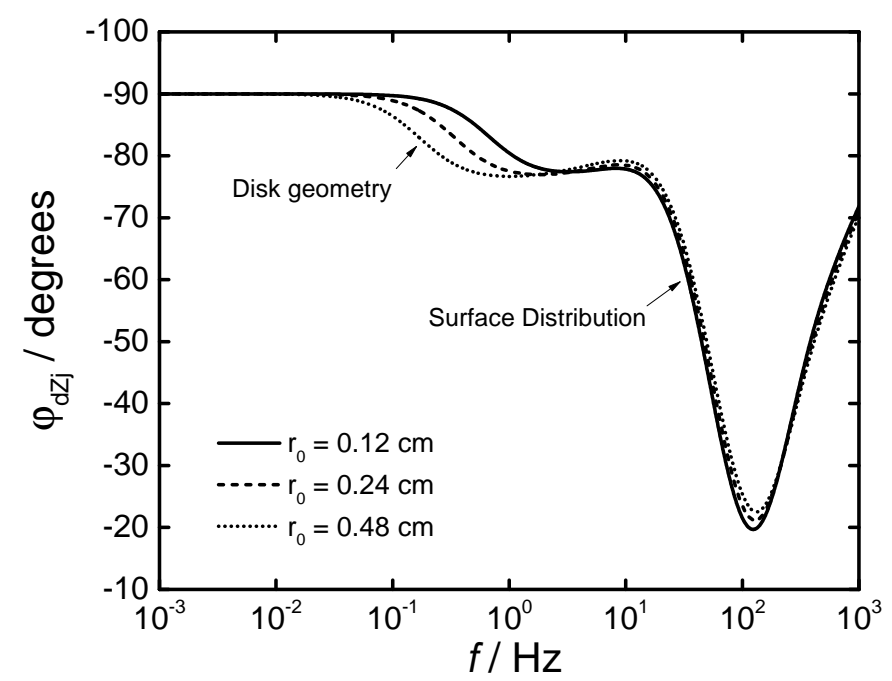

(a)

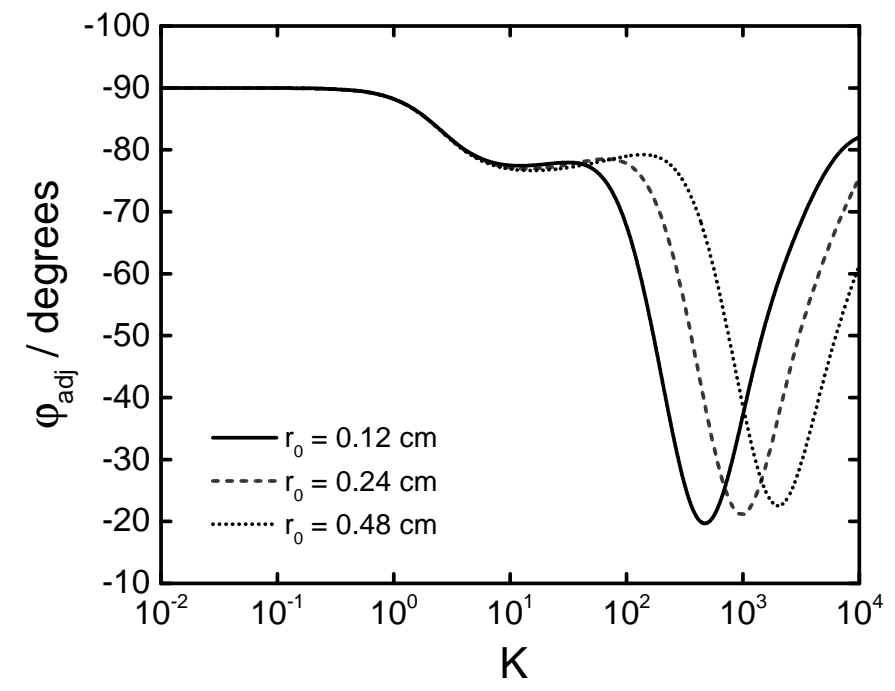

(b)

Figure 11: Imaginary-impedance-derived phase angles values calculated from the impedance data with the disk radius as a parameter: a) imaginary-impedance-derived phase angle as a function of frequency; b) imaginary-impedance-derived phase angle as a function of dimensionless frequency $K=\omega\left\langle C_{0}\right\rangle \pi r_{0} / 4 \kappa$. 


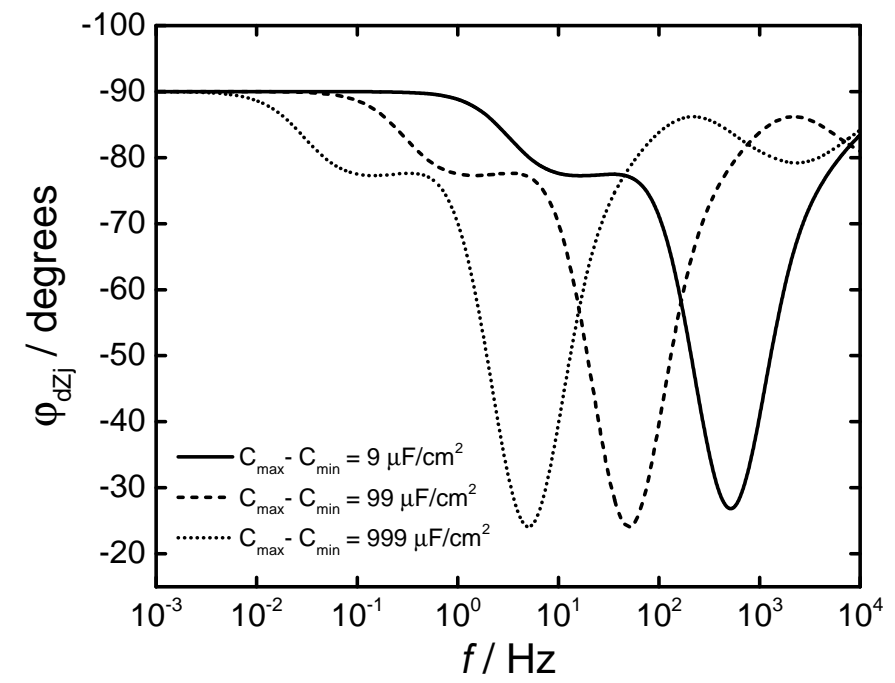

(a)

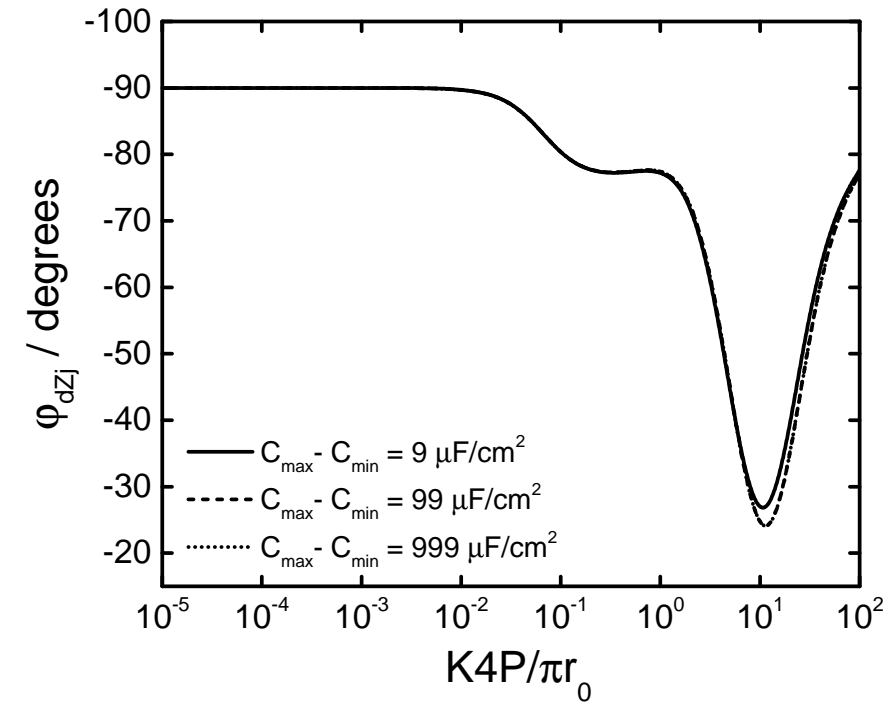

(b)

Figure 12: Imaginary-impedance-derived phase angle calculated from impedance data on disk electrodes with a radial distribution of capacitance with the amplitude of the square wave as a parameter: a) phase angle as a function of frequency; b) phase angle as a function of dimensionless frequency based on the averaged capacitance and the period of the square wave as the characteristic length. 


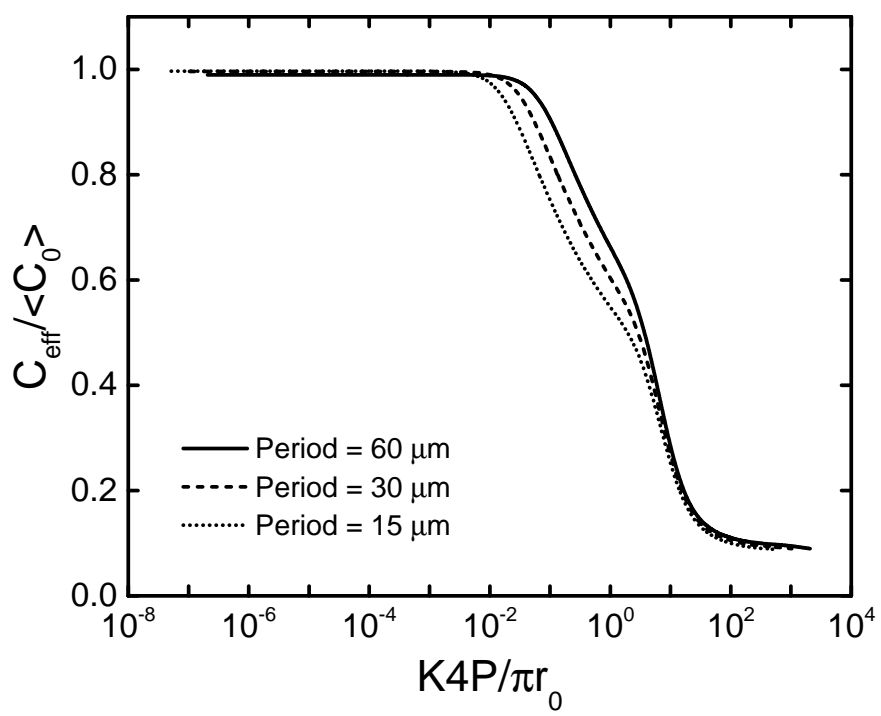

Figure 13: The ratio of the calculated effective capacitance and the surface-averaged input capacitance as a function of dimensionless frequency $K 4 P / \pi r_{0}$ with the period of the distribution as a parameter for disk electrodes within an insulating plane.

presented as a function of dimensionless frequency in Figure 13 with the period of the distribution as a parameter. In all cases, the simulated capacitance closely matched the averaged-surface capacitance of the electrode at low frequencies, indicated by a capacitance ratio value equal to 1 . At high frequencies, an initial decrease of the capacitance ratio was caused by the nonuniform current distribution on the disk surface. At a dimensionless frequency equal to one, a further decrease was observed due to the capacitance heterogeneity.

The characteristic frequency at which frequency dispersion occurs for a planar disk electrode exhibiting a distribution of capacitance may be expressed as

$$
f_{c, r_{0}}=\frac{2 \kappa}{\pi^{2}\left\langle C_{0}\right\rangle r_{0}}
$$




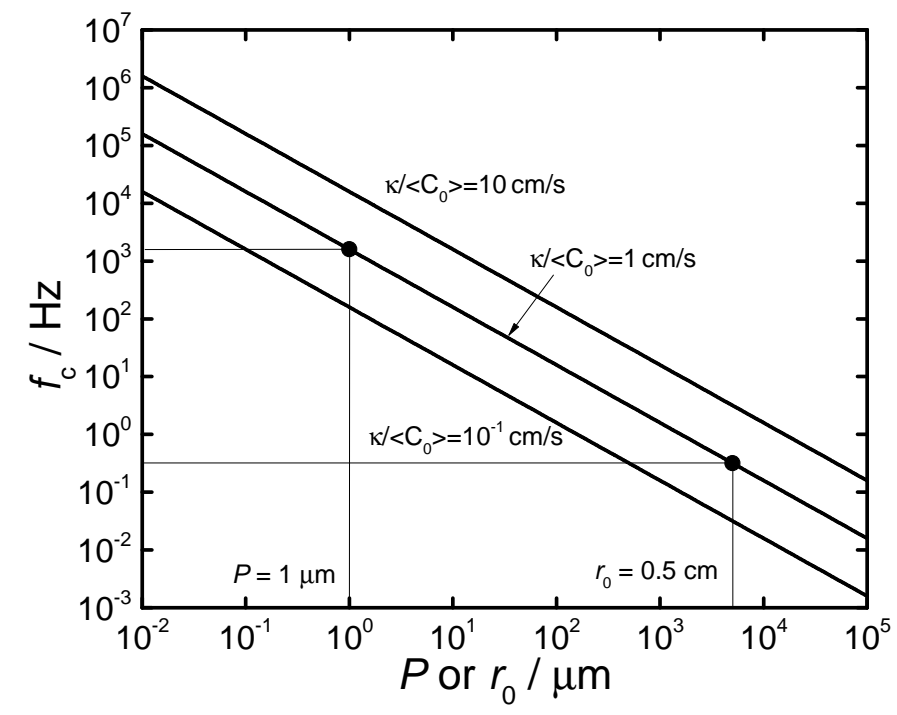

Figure 14: The frequency $K 4 P / \pi r_{0}=1$ at which the surface heterogeneity influences the impedance as a function of distribution period and disk radius with $\kappa /\left\langle C_{0}\right\rangle$ as a parameter.

where $\left\langle C_{0}\right\rangle$ represents the surface-averaged capacitance. The characteristic frequency at which frequency dispersion begins due to the capacitance distribution may be expressed as

$$
f_{c, P}=\frac{\kappa}{2 \pi\left\langle C_{0}\right\rangle P}
$$

in which the period of the distribution is the characteristic length associated with the distribution. The frequency at which dispersion begins is presented in Figure 14 as a function of both the disk radius as well as the period of the capacitance distribution with the ratio, $\kappa /\left\langle C_{0}\right\rangle$, as a parameter. The period of the distribution may be associated with the average width of grain sizes within a noncrystalline surface. For a $0.5 \mathrm{~cm}$ radius electrode in a system with $\kappa /\left\langle C_{0}\right\rangle=1 \mathrm{~cm} / \mathrm{s}$ (corresponding for example, to $\left\langle C_{0}\right\rangle=20$ $\mu \mathrm{F} / \mathrm{cm}^{2}$ and a $0.16 \mathrm{mM}$ sodium chloride concentration) and an average grain size of $1 \mu \mathrm{m}$, the frequency at which dispersion occurs due to the disk 
geometry is $400 \mathrm{mHz}$ while the frequency at which dispersion occurs due to the nonuniform capacitance is $1.6 \mathrm{kHz}$. The frequency dispersion due to the capacitance distribution will always occur at greater frequencies than the frequency dispersion due to the disk geometry.

\section{Conclusions}

The role of surface capacitance heterogeneity on solid electrodes was studied using numerical finite element simulations under the assumption of blocking electrode behavior. Multiple cell geometries including a recessed electrode, and an electrode within an insulating plane were used to assess the effect of a radial distribution of capacitance. It was shown that surface heterogeneity due to non-uniform capacitance only caused frequency dispersion at frequencies greater than $1 \mathrm{kHz}$ for a period of $1 \mu \mathrm{m}$.

Two distinct deviations from ideality were observed for a disk electrode in an insulating plane containing a periodic distribution of capacitance. The first is indicative of the effect of the disk geometry, and the second represents the influence of the surface heterogeneity. The characteristic length associated with a periodic distribution of capacitance is the period itself. Physically the period may represent the average width of grain sizes, which is usually on the order of microns. The characteristic frequency determined by the second deviation associated with capacitance distribution is $f_{c}=\kappa / 2 \pi\left\langle C_{0}\right\rangle P$ in which the period of the distribution is used as the characteristic length.

Surface heterogeneity due to a nonuniform distribution of capacitance does not provide a physical explanation for CPE behavior over a broad range of frequencies. A non-uniform capacitance only causes frequency dispersion at frequencies greater than those due to the geometry of a disk 
electrode contained within an insulating plane. The frequency dispersion due to the surface heterogeneity would only influence the impedance at frequencies lower than those influenced by the disk geometry if the period of the surface distribution were greater than the radius of the disk, which is physically impossible.

\section{Acknowledgements}

M. Orazem acknowledges partial support from the University of Florida Research Foundation Professorship.

\section{References}

[1] J.-B. Jorcin, M. E. Orazem, N. Pébère, B. Tribollet, CPE analysis by local electrochemical impedance spectroscopy, Electrochimica Acta 51 (2006) 1473-1479.

[2] B. Hirschorn, M. E. Orazem, B. Tribollet, V. Vivier, I. Frateur, M. Musiani, Constant-phase-element behavior caused by resistivity distributions in films: 1. theory, Journal of The Electrochemical Society 157 (2010) C452-C457.

[3] B. Hirschorn, M. E. Orazem, B. Tribollet, V. Vivier, I. Frateur, M. Musiani, Constant-phase-element behavior caused by resistivity distributions in films: 2. applications, Journal of The Electrochemical Society 157 (2010) C458-C463.

[4] M. E. Orazem, B. Tribollet, V. Vivier, S. Marcelin, N. Pébère, A. L. Bunge, E. A. White, D. P. Riemer, I. Frateur, M. Musiani, Dielectric properties of materials showing constant-phase-element (CPE) 
impedance response, Journal of The Electrochemical Society 160 (2013) C215-C225.

[5] E. A. White, M. E. Orazem, A. L. Bunge, Characterization of damaged skin by impedance spectroscopy: Chemical damage by dimethyl sulfoxide, Pharmaceutical Research 30 (2013) 2607-2624.

[6] M. Musiani, M. Orazem, N. Pébère, B. Tribollet, V. Vivier, Determination of resistivity profiles in anti-corrosion coatings from constantphase-element parameters, Progress in Organic Coatings 77 (2014) 2076-2083.

[7] V. M. W. Huang, V. Vivier, M. E. Orazem, N. Pébère, B. Tribollet, The apparent CPE behavior of an ideally polarized disk electrode: A global and local impedance analysis, Journal of The Electrochemical Society 154 (2007) C81-C88.

[8] T. Borisova, B. Ershler, Determination of the zero voltage points of solid metals from measurements of the capacity of the double layer, Zhurnal Fizicheskoi Khimii 24 (1950) 337-344.

[9] C. L. Alexander, B. Tribollet, M. E. Orazem, Contribution of surface distributions to constant-phase-element (cpe) behavior: 1. influence of roughness, Electrochimica Acta (2015).

[10] R. Leek, N. A. Hampson, The dispersion of double-layer capacitance with frequency i. smooth solid electrodes, Surface Technology 7 (1978) $151-155$.

[11] G. J. Brug, A. L. G. van den Eeden, M. Sluyters-Rehbach, J. H. Sluyters, The analysis of electrode impedances complicated by the pres- 
ence of a constant phase element, Journal of Electroanalytical Chemistry 176 (1984) 275-295.

[12] T. Pajkossy, L. Nyikos, Impedance of planar electrodes with scaleinvariant capacitance distribution, Journal of Electroanalytical Chemistry 332 (1992) 55-61.

[13] B. Kurtyka, R. de Levie, Frequency dispersion associated with a non-homogeneous interfacial capacitance, Journal of Electroanalytical Chemistry 322 (1992) 63-77.

[14] C. F. C. M. Fong, D. D. Kee, P. N. Kalomi, Advanced Mathematics for Applied and Pure Sciences, Gordon and Breach Science Publishers, Amsterdam, The Netherlands, 1997.

[15] J. S. Newman, Resistance for flow of current to a disk, J. Electrochem. Soc. 113 (1966) 501-502. 